\title{
REDUCTIONS OF $n$-FOLD COVERS ${ }^{1}$
}

\author{
SAUL STAHL
}

\begin{abstract}
Motivated by L. Lovász's recent proof of the Kneser conjecture [3], this paper offers another result which relates topological and graph theoretical concepts. A method for converting $n$-fold covers to $(n-1)$-fold covers is presented. This yields a strengthening of the classical Borsuk, Lusternik and Schnirelmann theorem on covers of spheres. The same conversion also has applications to multicolorings of graphs.
\end{abstract}

Let $\mathcal{S}=\left\{S_{i}\right\}_{i=1}^{m}$ be a family of subsets of the set $S$. We say that $\mathcal{S}$ is an $n$-fold cover of $S$ if every element of $S$ is contained in at least $n$ of the $S_{i}$. The letters $m$ and $n$ always denote positive integers.

A classical theorem of Borsuk [1] and of Lusternik and Schnirelmann [4] states that in any cover of the $k$-dimensional sphere by means of $k+1$ closed subsets, at least one of the subsets contains a pair of antipodal points. It is well known that covers employing $k+2$ subsets exist none of whose members contain antipodal points. Examples have been constructed by Borsuk [1] and Gale [2]. The author is indebted to the referee for suggesting the following easily described example. Inscribe a regular $(k+1)$ dimensional simplex inside $S^{k}$ in $\mathbf{R}^{k+1}$. Surround each of the $k+2$ vertices by a $k$-dimensional disk in $S^{k}$, centered at the vertex and large enough so that it is not quite a hemisphere. These $k+2$ closed disks are obviously a cover of $S^{k}$, but none of them contains an antipodal pair of points. However, Corollary 1 to Theorem 1 shows that the classical theorem can be strengthened to an "antipodal points result" for covers of $S^{k}$ by more than $k+1$ subsets. The basic idea is to add a requirement on the number of times each point is covered.

THEOREM 1. Let $\mathcal{S}=\left\{S_{i}\right\}_{i=1}^{m}$ be an $n$-fold cover of the set $S$, with $m>n$. For each $i \in\{1,2, \ldots, m-2\}$ define

$$
T_{i}=\left[\bigcap_{j=1}^{i+1} S_{j}\right] \cup\left[S_{i+2} \cap\left(\bigcup_{j=1}^{i+1} S_{j}\right)\right] .
$$

Then the family $\mathcal{T}=\left\{T_{i}\right\}_{i=1}^{m-2}$ has the following properties:

(a) $\mathcal{T}$ is an $(n-1)$-fold cover of $S$;

(b) if any two elements of $S$ are both contained in some member of $\mathcal{T}$ then

Received by the editors October 27, 1977 and, in revised form, February 27, 1978.

AMS (MOS) subject classifications (1970). Primary $05 \mathrm{Cl} 0$.

${ }^{\mathrm{I}}$ The author would like to thank Dr. Fred Galvin for many helpful conversations in this context. 
they are also both contained in some member of $\mathcal{S}$.

Proof. Let $x$ be any element of $S$. Since $\mathcal{S}$ is an $n$-fold cover of $S$, there exist integers $1 \leqslant i_{1}<i_{2}<\cdots<i_{n} \leqslant m$ such that $x \in \cap_{j=1}^{n} S_{i}$. If $i_{1}>1$, then clearly $x \in S_{i} \cap\left(\cup_{j=1}^{i-1} S_{j}\right)$ for all $i \in\left\{i_{2}, \ldots, i_{n}\right\}$ and consequently $x$ is contained in all the $n-1$ sets $T_{i}$ where $i \in\left\{i_{2}-2, \ldots, i_{n}-2\right\}$. If $i_{1}=1$, let $k$ be the largest integer such that $i_{k}=k$. Note that $i_{j}=j$ for $j \in$ $\{1,2, \ldots, k\}$ and that $i_{j} \geqslant j+1$ for $j \geqslant k+1$. Since $x \in \cap_{j=1}^{i} S_{j}$ for $i \in\{1,2, \ldots, k\}$ it follows that $x \in T_{i}$ for $i \in\{1,2, \ldots, k-1\}$. Observe that as $m>n$ we may conclude that $m-2 \geqslant n-1 \geqslant k-1$ and so we do indeed have here $k-1$ members of $\mathcal{T}$ which contain $x$. For $j \geqslant k+1$ we know that $i_{j}>i_{1}$ and hence $x \in \cup_{i=1}^{i_{i}-1} S_{i}$. Since $x \in S_{i_{j}}$ we conclude again that $x \in T_{i}$ for $i \in\left\{i_{k+1}-2, \ldots, i_{n}-2\right\}$. From the definition of $k$ it follows that the sets $\{1,2, \ldots, k-1\}$ and $\left\{i_{k+1}-2, \ldots, i_{n}-2\right\}$ are disjoint and so $x$ is again contained in $n-1$ of the $T_{i}$.

(b) Suppose $x$ and $y$ are both in $T_{i}$ for some $i \in\{1,2, \ldots, m-2\}$. If $x$ and $y$ are both in $\bigcap_{j=1}^{i+1} S_{j}$ or both in $S_{i+2} \cap\left(\cup_{j=1}^{i+1} S_{j}\right)$, then $x$ and $y$ are both in $S_{1}$ or both in $S_{i+2}$, respectively. Hence there only remains to be considered the case where $x \in \bigcap_{j=1}^{i+1} S_{j}$ and $y \in S_{i+2} \cap\left(\cup_{j=1}^{i+1} S_{j}\right)$. But then $x$ and $y$ are both in the same $S_{i}$ for some $i \in\{1,2, \ldots, i+1\}$. Thus there always does exist an $S_{i}$ containing both $x$ and $y$. Q.E.D.

It should be noted here that the hypothesis $m>n$ only excludes the trivial cover wherein each member of $\delta$ actually equals $S$. In that case the conclusion of the theorem does not hold, but the situation is clear and uninteresting.

Corollary 1. Given two integers $n \geqslant 1$ and $k \geqslant 0$, let $\mathcal{S}=\left\{S_{i}\right\}_{i=1}^{2 n+k-1}$ be an $n$-fold cover of the $k$-dimensional sphere $S^{k}$. If each $S_{i}$ is a closed subset, then some member of $\mathcal{S}$ contains an antipodal pair of points of $S^{k}$.

Proof. By induction on $n$. If $n=1$, then this is the above mentioned theorem of Borsuk, Lusternik and Schnirelmann. Now assume the validity of this corollary for every $(n-1)$-fold cover of $S^{k}$ (where $k$ is fixed) with closed subsets, and let $\delta$ be an $n$-fold such cover. If $2 n+k-1 \leqslant n$, then necessarily $n=1, k=0$, and the corollary follows trivially. Else, Theorem 1 applies. Let $\mathcal{T}$ be the $(n-1)$-fold cover constructed from $\mathcal{S}$. It is clear from the definition of the $T_{i}$ that they are closed subsets, and since $\mathcal{T}$ contains $2 n+k-1-2=2(n-1)+k-1$ members, it follows from the induction hypothesis that some $T_{i}$ contains an antipodal pair of points. Part (b) of Theorem 1 implies that this antipodal pair is also contained in some $S_{j}$. Q.E.D.

D. Gale [2] has constructed for $n, k$ as above, an $n$-fold cover of $S^{k}$ which consists of $2 n+k$ open hemispheres. Since these can be easily trimmed down to closed subsets without ceasing to be an $n$-fold cover, it may be said that Corollary 1 is a best possible result. 
Theorem 1 can also be used to give an alternate proof of a graph theoretical theorem first proved in Stahl [5]. An independent subset of the graph $G$ is a set of vertices no two of which are adjacent. The nth chromatic number of $G, \chi_{n}(G)$, is the least number of sets in any $n$-fold covering of $G$ with independent subsets.

COROLlaRY 2. If the graph $G$ contains an edge, then

$$
\chi_{n}(G) \geqslant 2+\chi_{n-1}(G) \text { for } n=2,3,4, \ldots
$$

Proof. Let $\mathcal{S}=\left\{S_{i}\right\}_{i=1}^{m}$ be an $n$-fold cover of $G$ with independent subsets, where $m=\chi_{n}(G)$. Since $G$ does contain an edge, none of the $S_{i}$ equals $G$, and hence $m>n$. Again Theorem 1 applies and we let $\mathcal{T}$ be the resulting $(n-1)$-fold cover of $G$. If some $T_{i}$ were not independent it would contain a pair of adjacent vertices of $G$. Because of property (b), however, such a pair would necessarily be contained in some $S_{j}$ as well, thus contradicting the independence of the members of $\delta$. Consequently each member of $\mathcal{T}$ is independent and hence $\chi_{n-1}(G) \leqslant m-2=\chi_{n}(G)-2$. Q.E.D.

\section{BIBLIOGRAPHY}

1. K. Borsuk, Drei Sätze über die n-dimensionale euklidische Sphäre, Fund. Math. 20 (1933), $177-190$.

2. D. Gale, Neighboring vertices on a convex polyhedron, Linear inequalities and related systems, Ann. of Math. Studies, no. 38, Princeton Univ. Press, Princeton, N.J., 1956, pp. 255-263.

3. L. Lovász, Kneser's conjecture, homotopy and Borsuk's theorem, J. Combinatorial Theory Ser. B (to appear).

4. L. Lusternik and L. Schnirelmann, Méthodes topologiques dans les problèmes variationnels, Gosudarstvennoe Izdat., Moscow, 1930; rev. French transl., Actualités Sci. Indust., no. 118, Hermann, Paris, 1934.

5. S. Stahl, $n$-tuple colorings and associated graphs, J. Combinatorial Theory Ser. B 20 (1976), 185-203.

Department of Mathematics, University of Kansas, Lawrence, Kansas 66045 